\title{
Research Paper \\ Comparison of Motor Skills and Postures of Elite Male Teenage Volleyball and Basketball Players
}

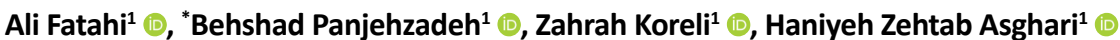

1. Department of Sports Biomechanics, Faculty of Physical Education and Sports Science, Central Tehran Branch, Azad University, Tehran, Iran.

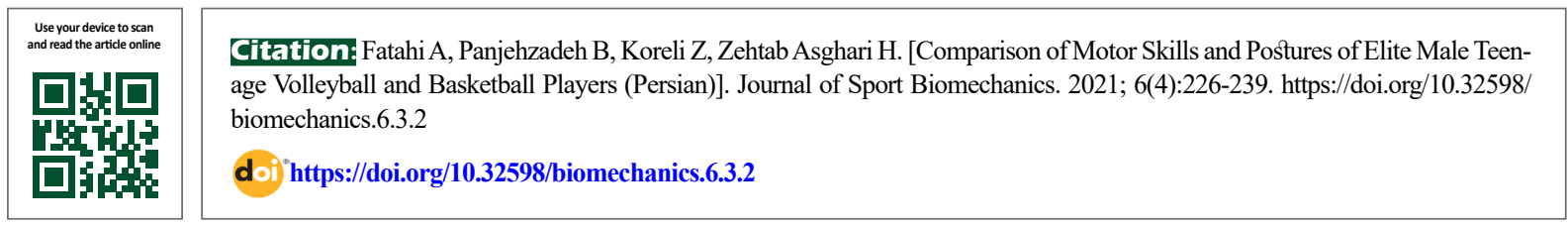

\section{(1) $\$$}

Article Info:

Received: 23 Aug 2020 Accepted: 28 Oct 2020 Available Online: 01 Mar 2021

Keywords:

Motor skills, Posture, Teenage boys, Volleyball, Basketball

\section{ABSTRACT}

Objective To achieve the excellent levels of athletic performance, professional athletes need to do continuous exercises and strengthen special muscles of the body and have to spend a lot of time training in that sport. As a result, depending on the prevailing condition of each sport, the physical orientation and the degree of abnormalities of the athletes in that sport are affected.

Methods The current study is descriptive and cross-sectional. The statistical population was 12-14 years old boys in middle and high school. The statistical sample of this study included 30 people (13 volleyball players and 17 basketball players) with a targeted training rate of at least three sessions per week. The Chippaux Smirak Index (CSI) method was used to evaluate the arch of the foot and to determine the angles and degrees of kyphosis and lordosis in radiographs. Independent t-test and Mann-Whitney test were used to compare the variables of motor skills and posture by SPSS V. 18 at a significant level of 0.05 . Results The results of independent t-test to compare the variables of motor skills in the two groups of volleyball and basketball show that there is a significant difference between the two groups in the Sargent test and $4 \mathrm{mX9m}(P=0.000)(P \geq 0.05)$. The results were different in strength test $(P=0.41)$ and Situp $(P=0.75)$ so that there was no significant difference between the two groups $(P \geq 0.05)$. The results of Mann-Whitney test to compare posture-related variables showed that there was no significant difference between the two groups in any of the parameters of varus, valgus, pronation, supination, lordosis, kyphosis, foot flat $(P \geq 0.05)$.

Conclusion The results showed that volleyball players have more impressive and explosive power than basketball players, but in terms of muscle strength and endurance, there is not much difference between these two groups, which is probably due to the nature and specific characteristics of their sports. Therefore, it seems necessary to pay more attention to athletes' training programs to improve their motor skills and physical condition. In this study, the behavior and common habits of the subjects in interaction with the environment and hereditary characteristics have not been studied, so more research is needed in the etiology of musculoskeletal abnormalities.

\section{* Corresponding Author:}

\section{Behshad Panjehzadeh, PhD. Candidate}

Address: Department of Sports Biomechanics, Faculty of Physical Education and Sports Science, Central Tehran Branch, Azad University, Tehran, Iran. Tel: +98 (918) 3323922

E-mail: behshadpanjehzadeh@gmail 


\section{Extended Abstract}

\section{Introduction}

$\mathbf{H}$

ealth, as the most important human asset, is a positive aspect of hygiene. This essential human asset includes social, spiritual, emotional, mental, communicative, and physical dimensions. The physical dimension of health (i.e. the desired physical condition in human life) is of particular importance, and its positive and negative changes can affect other aspects of human life [1]. The quality of adolescents' motor development is an important issue that experts have always considered in physical and motor development. In this regard, basic motor skills are among the characteristics that provide a framework for motor and sports skills in late adolescence, leading to a better quality of daily life activities later in life.

In stature abnormalities, therapeutic intervention is performed to maintain and achieve proper body mechanics. The goal of correctional programs is to identify, educate, prevent, and improve abnormalities. The first step in identifying the abnormality is to pay attention to the person's posture and limbs because all musculoskeletal disorders, regardless of the primary cause, manifest their symptoms on the person's posture.

"Posture" is the skeletal and muscular balance that protects the body's supporting structures against injury and progressive deformity at work or rest. In such a situation, the muscles are most efficient, and the abdomen and chest organs are in good condition [4]. Improper posture puts pressure on the intervertebral discs and increases tension in the spine's posterior tissues [6]. If the low back's curvature is not maintained and the pelvis is not in the proper vertical position, it causes posterior pelvic tilt, and in the long run, causes changes in the lumbar spine [7].

The ability to maintain postural control is an essential factor in performing many daily activities, and motor skills play a significant role in children's learning ability and provide the basis for the development of other essential learnings such as academic and social skills $[8,9]$; as a result, any disruption in the motor skills process causes weakness and problems in the academic, social, learning and individual skills of adolescents [10]. During the developmental stages, motor skills significantly impact motor development throughout life because they allow for better growth by exploring the environment more.

The development of basic skills is the basis of children's motor development. A variety of movement experiences can provide children with the correct information to develop their perception of the world around them.

Motor skills are broken up into two categories: first, fine motor skills, which include directional, distinct, precise, and skillful movements that require the use of smaller and delicate muscle groups; second, the gross motor skills performed by the large muscles of the body, which include general movement, statics, and balance control [11].

Due to the lack of adequate knowledge and education in adolescents (middle and high school ages), the prevalence of stature abnormalities is common among students [13], while little has been done on elite athletes. On the other hand, anthropometry is used as a suitable tool in biological developmental research, and from the perspective of sports experts, it is an important but controversial indicator for studying the development of motor skills [14]. Regarding musculoskeletal abnormalities, especially in the lower limbs (such as flat sole, hollow sole, knock-knee, bowleg, lordosis, and kyphosis), the question has always been whether these factors affect motor skills during adolescence or not. This study aimed to investigate the motor skills and postures of adolescents concerning their sports activities.

\section{Methods}

This research was a cross-sectional descriptive study, and its statistical population consisted of adolescents aged 1214 years in middle and high school. This study's statistical sample included 30 people (13 volleyball players and 17 basketball players) with at least three training sessions per week, and their selection was purposive.

Before conducting the research, while explaining the necessity and importance of the research to the subjects, a consent form for taking radiographs and another written consent form was taken from all subjects' parents. Movement motor skills included "grip strength", "sit-ups", "Sargent jump", and "agility ( $4 \mathrm{mX} 9 \mathrm{~m})$ test", the total scores of which were calculated for each person.

Demographic information of the subjects in the volleyball group (including age $12.92 \pm 0.86$ years, height $169.23 \pm 4.34$ $\mathrm{cm}$, and weight $51.69 \pm 3.19 \mathrm{~kg}$ ) and in the basketball group (including age $12.82 \pm 0.88$ years, height $166.24 \pm 4.53$, and weight $56.35 \pm 6.27 \mathrm{~kg}$ ) were measured. Independent t-test and Mann-Whitney test were used to compare the variables of motor skills and posture by SPSS software at a significance level of 0.05 . In this study, the CSI method was used to evaluate the arch of the sole. Test items included: grip strength, sit-ups, Sargent jumps, and agility ( $4 \mathrm{mX} 9 \mathrm{~m})$ test. 
Table 1. Results of independent t-test to compare the variables of motor skills of the subjects in the "volleyball" and "basketball" groups

\begin{tabular}{|c|c|c|c|c|c|c|}
\hline Variables & Group & Mean $\pm S D$ & Mean Difference & $\mathbf{F}$ & T & $\mathbf{P}$ \\
\hline Grip strength (paw) (kg) & $\begin{array}{l}\text { Volleyball } \\
\text { Basketball }\end{array}$ & $\begin{array}{l}0.52 \pm 0.06 \\
0.47 \pm 0.8\end{array}$ & 1.52 & 5.73 & 0.84 & 0.41 \\
\hline Sit-up (number) & $\begin{array}{l}\text { Volleyball } \\
\text { Basketball }\end{array}$ & $\begin{array}{c}43.07 \pm 7.74 \\
45.17 \pm 11.14\end{array}$ & -1.09 & 4.49 & -0.31 & 0.75 \\
\hline Sargent jump (cm) & $\begin{array}{l}\text { Volleyball } \\
\text { Basketball }\end{array}$ & $\begin{array}{l}70.61 \pm 8.58 \\
73.00 \pm 6.98\end{array}$ & 20.61 & 1.21 & 0.28 & 0.00 \\
\hline Agility $(4 \mathrm{~m} \times 9 \mathrm{~m})$ test (time) & $\begin{array}{l}\text { Volleyball } \\
\text { Basketball }\end{array}$ & $\begin{array}{l}8.86 \pm 3.45 \\
9.74 \pm 9.73\end{array}$ & 1.59 & 0.01 & 0.89 & 0.00 \\
\hline
\end{tabular}

To perform the test, the researcher thoroughly explained to the subjects how to perform each skill; then, the test was presented for each athlete, and he was asked to perform each skill after one observation. To determine the angles and degrees of kyphosis (angles VP-T12, VP-ITL ${ }^{1}$, ICT-ITL ${ }^{2}$ ) and lordosis (angles DM-T12, DM-ITL, ILS ${ }^{3}$-ITL) in radiographs, the Cobb angle measurement method was used.

\section{Results}

The results of the independent t-test to compare the variables of motor skills in the two groups of volleyball and basketball confirm that there was a significant difference between the two groups in the Sargent test and the agility $(4 \mathrm{mX} 9 \mathrm{~m})$ test $(\mathrm{P}=0.00)(\mathrm{P} \geq 0.05)$. The results of the grip strength test $(\mathrm{P}=0.41)$ and sit-ups $(\mathrm{P}=0.75)$ were different, but there was no significant difference between the two groups $(\mathrm{P} \geq 0.05)$.

Mann-Whitney test results to compare posture-related variables indicated no significant difference between the two groups in any of the parameters of varus, valgus, pronation, supination, lordosis, kyphosis, hollow sole, and flat sole $(\mathrm{P} \geq 0.05)$ (Tables 1 and 2 ).

\section{Discussion and Conclusion}

This study compared selected motor skills including "grip strength" (paw), "sit-ups", "Sargent jump", "agility $(4 \mathrm{mX} 9 \mathrm{~m})$ test", and "posture" of elite adolescent volley-

\footnotetext{
1. Vertebra prominens

2. Inclination cervical thoracic-inclination thoracic lumbar

3. Inclination lumbar sacral
}

ball and basketball players. This study proved that sports, due to activating the muscles, can adequately control stature condition, but motor skills, depending on the nature of each sport, had a higher impact on some parameters. For instance, this study discovered that volleyball players had more impressive explosive power.

Motor skills are a prerequisite for learning special sports skills such as athletics, basketball, volleyball, etc. Numerous factors such as individual differences, musculoskeletal abnormalities, environmental conditions, and gender differences can help develop these skills. However, based on the results and due to the subjects' abnormalities, their training patterns could be the most crucial cause of the abnormalities.

Since more physical activity required more training sessions and because all of the athletes were elite, one of the reasons for the increase in some abnormalities might be the number of sessions and training duration $[19,20]$. On the other hand, insisting on using a particular group of specific muscles specific to the desired sport and not paying attention to muscles that are not effective in performing techniques may also cause mechanical and structural disorders and create a physically undesirable situation.

\section{Ethical Considerations}

\section{Compliance with ethical guidelines}

All ethical principles are considered in this article. The participants were informed of the purpose of the research and its implementation stages. They were also assured about the confidentiality of their information and were free to leave the study whenever they wished, and if desired, the research results would be available to them. 
Table 2. Mann-Whitney test results to compare the subjects' postural variables in "volleyball" and "basketball" groups

\begin{tabular}{|c|c|c|c|c|c|}
\hline Indexes & Groups & Mean \pm SD & Mean Rank & $z$ & $\mathbf{P}$ \\
\hline \multirow{3}{*}{ Varus } & Volleyball & $1.38 \pm 1.3$ & 17.73 & \multirow{3}{*}{-1.28} & \multirow{3}{*}{0.119} \\
\hline & & & & & \\
\hline & Basketball & $0.76 \pm 0.90$ & 13.79 & & \\
\hline \multirow{3}{*}{ Valgus } & Volleyball & $0.53 \pm 1.33$ & 15.35 & \multirow{3}{*}{-0.12} & \multirow{3}{*}{0.893} \\
\hline & & & & & \\
\hline & Basketball & $0.58 \pm 1.32$ & 15.62 & & \\
\hline \multirow{3}{*}{ Pronation } & Volleyball & $0.53 \pm 0.66$ & 14.35 & \multirow{3}{*}{-0.69} & \multirow{3}{*}{0.483} \\
\hline & & & & & \\
\hline & Basketball & $0.70 \pm 0.68$ & 16.38 & & \\
\hline \multirow{3}{*}{ Supination } & Volleyball & $0.61 \pm 0.65$ & 18.42 & \multirow{3}{*}{-1.92} & \multirow{3}{*}{0.051} \\
\hline & & & & & \\
\hline & Basketball & $0.23 \pm 0.56$ & 13.26 & & \\
\hline \multirow{3}{*}{ Lordosis } & Volleyball & $0.30 \pm 0.48$ & 14.12 & \multirow{3}{*}{-0.88} & \multirow{3}{*}{0.327} \\
\hline & & & & & \\
\hline & Basketball & $0.47 \pm 0.51$ & 16.56 & & \\
\hline \multirow{3}{*}{ Kyphosis } & Volleyball & $0.69 \pm 0.75$ & 18.38 & \multirow{3}{*}{-1.85} & \multirow{3}{*}{0.016} \\
\hline & & & & & \\
\hline & Basketball & $0.23 \pm 0.43$ & 13.29 & & \\
\hline \multirow{3}{*}{ Hollow sole } & Volleyball & $0.15 \pm 0.37$ & 16.31 & \multirow{3}{*}{-0.84} & \multirow{3}{*}{0.359} \\
\hline & & & & & \\
\hline & Basketball & $0.05 \pm 0.24$ & 14.88 & & \\
\hline \multirow{3}{*}{ Flat sole } & Volleyball & $0.46 \pm 0.51$ & 17.46 & \multirow{3}{*}{-1.32} & \multirow{3}{*}{0.618} \\
\hline & & & & & \\
\hline & Basketball & $0.29 \pm 0.68$ & 14.00 & & \\
\hline
\end{tabular}

Funding

This research did not receive any grant from funding agencies in the public, commercial, or non-profit sectors.

Authors' contributions

All authors equally contributed to preparing this article.

Conflicts of interest

The authors declared no conflict of interest.

Acknowledgements

The authors would like to thank all of the Aesthetic \& Clinical Center, Pouyesh Setaregan Salamat, Tehran. 
This Page Intentionally Left Blank 


\title{
مقايسه مهارتهاى حركتى و ياسجر يسران نوجوان واليباليست و بسكتباليست نخبه
}

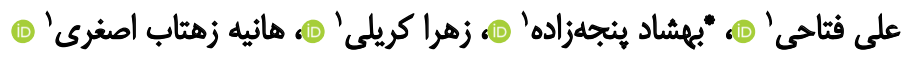 \\ ا. كروه بيومكانيك ورزشى، دانشكده تربيت بدنى و علوم ورزشى، دانشكاه آزاد اسلامى، واحد تهران مركزى، تهران، ايران.
}

\begin{abstract}
حكיد

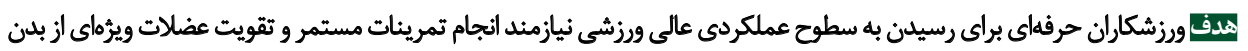

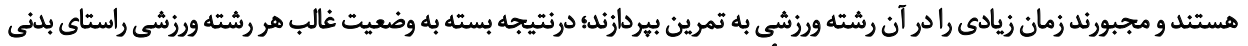

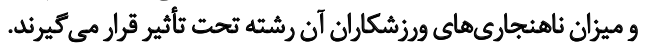

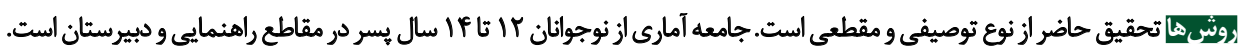

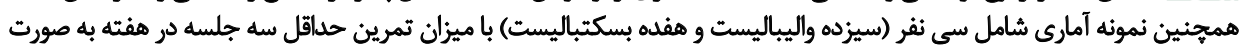

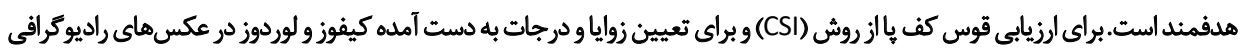

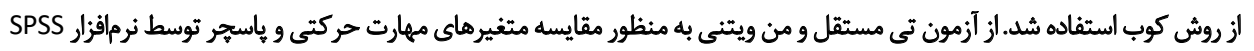

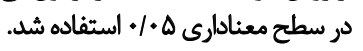

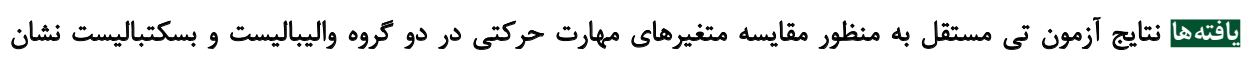

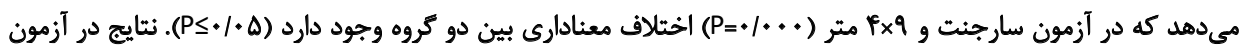

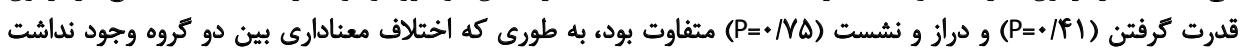

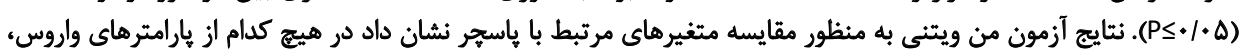

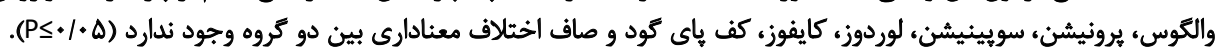

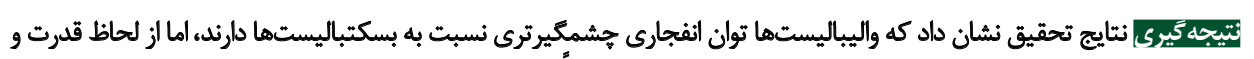

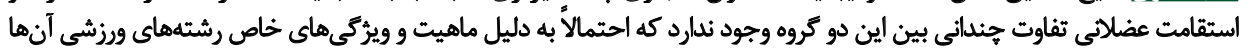

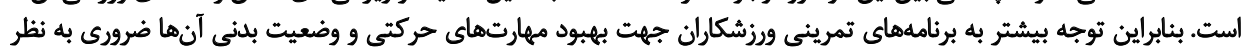

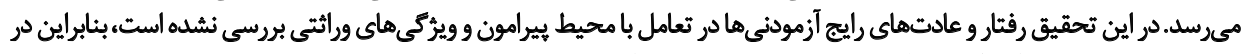

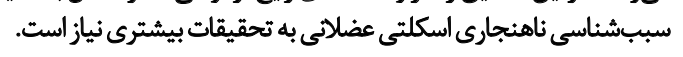

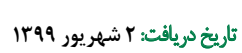

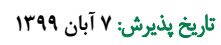

كيفيت بهترى از فعاليتهاى روزانه زندكى رابه همراه آورند.

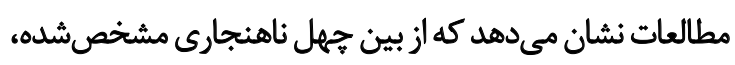

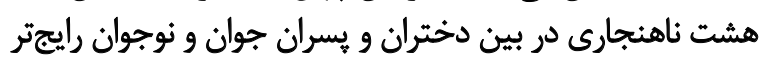

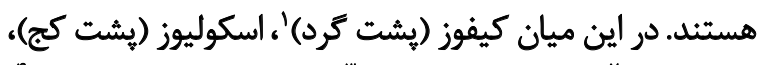

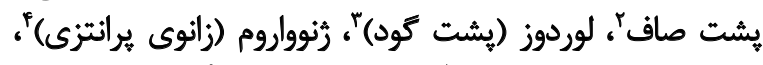

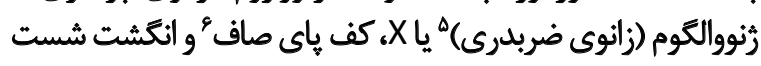

1. Thoracic kyphosis

2. Flat back

3. Kypholordotic posture

4. Genu varum

5. Genus valgum

6. Flat foot

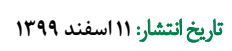

كليدواروها:

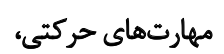

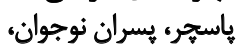
واليبال، بسكتبال نوجرال

\section{.}

تندرستى مهمهرين سرمايه انسان است. اين سرمايه مهمه شامل

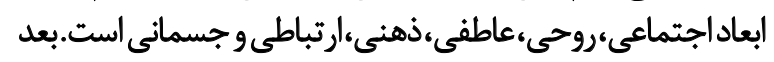

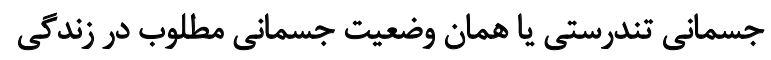

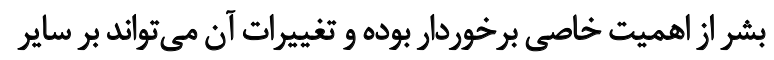

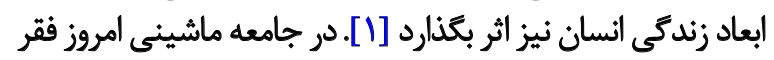

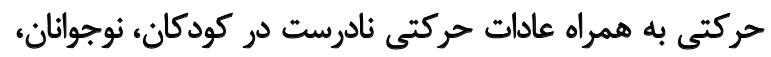

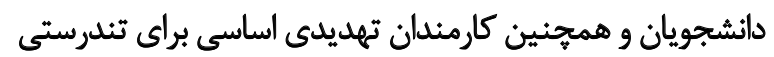

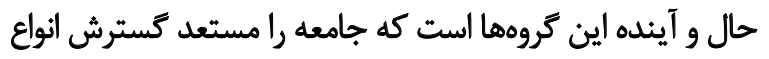

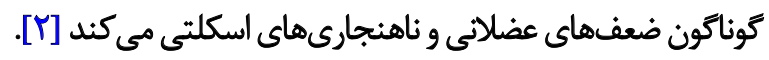

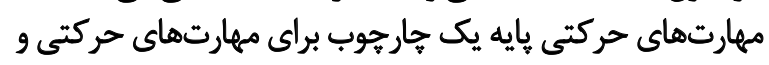
ورزشى در سنين اواخر نوجوانى هستند كه در ادامه عمر مي توانئ براند

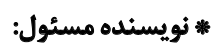
دكتر بهشاد بنجهةزاده نشانى: تيهران، دانشعاه آزاد اسلامى، واحد تهران مركزى، دانشكده تربيت بدنى و علوم ورزشى، كروه بيومكانيك ورزشى.

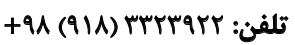
يست الكترونيكى: behshadpanjehzadeh@gmail 
تركيب الكوهاى حركتى بنيادى مي تواند حركات بيجيجيدهتر ورزشيى

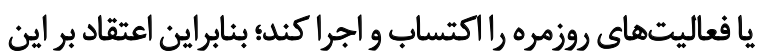

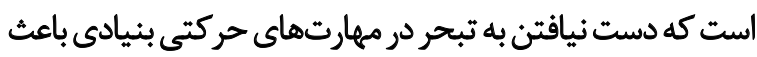

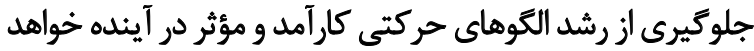

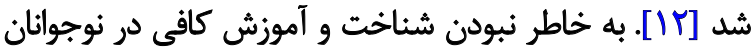

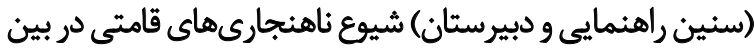

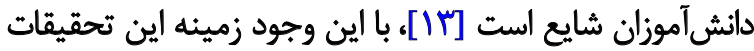

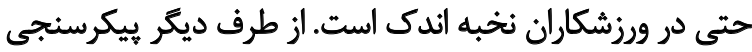

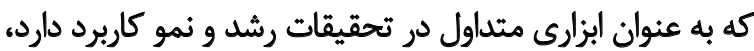

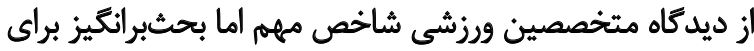

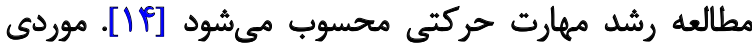

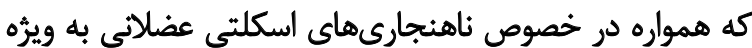

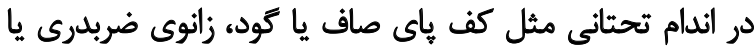

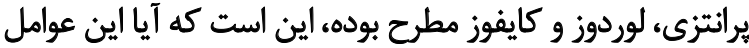

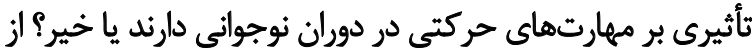

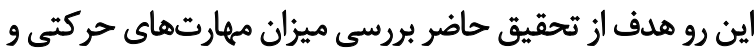

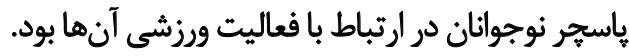

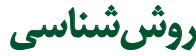

تحقيق حاضر از نوع توصيفى و مقطعى است. جامعه آمارى

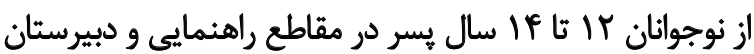

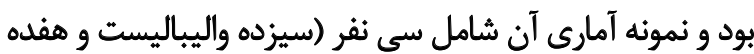

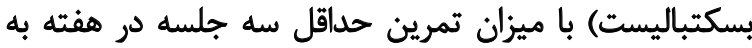

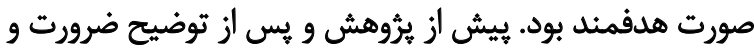

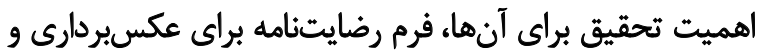

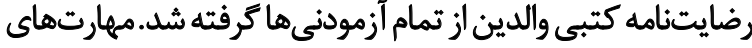

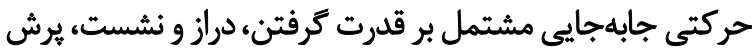

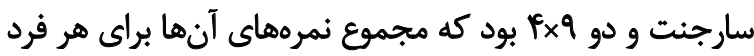

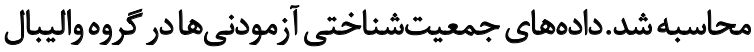

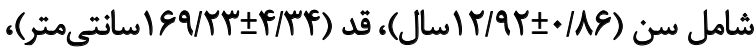

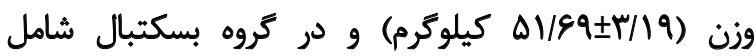

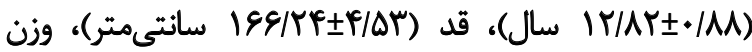

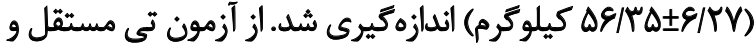

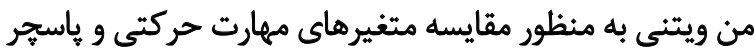
توسط نرمافزار SPSS در سطح معنادارى ه ه • • استفاده شد.

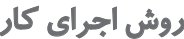

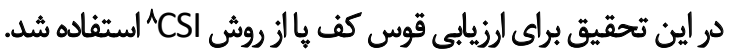

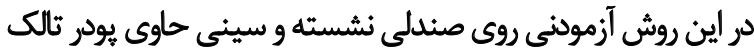

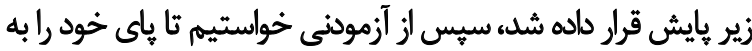

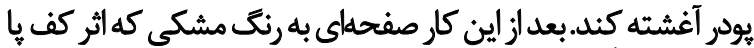

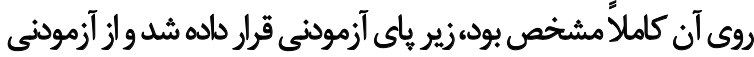
خواسته شد تا روى ياهايش بايستد و سر خود را بالانكَه دارد و دوباره
كج (هالوكس والكوس) " رامىتوان رايجترين ناهنجارىها نام برد

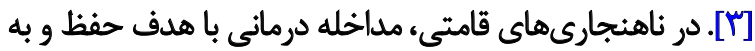

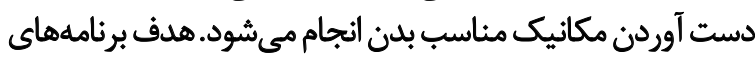

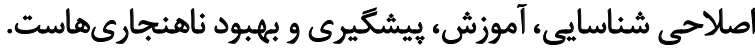

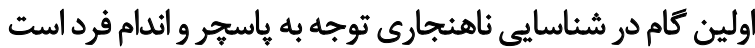

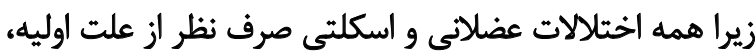
علايم خود را بر ياسجر فرد بروز مى دهند.

ياسجر عبارت است از تعادل اسكلتى وعضلاتى كه ساختمانهاى

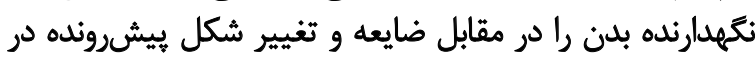

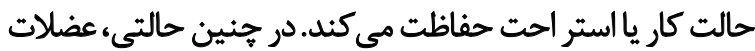

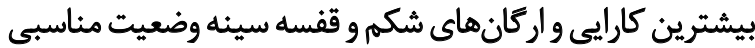

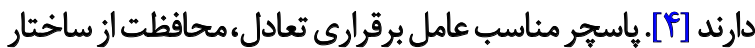

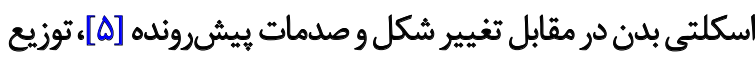

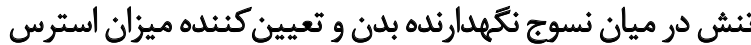

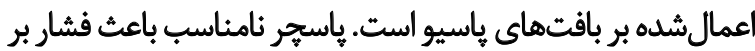

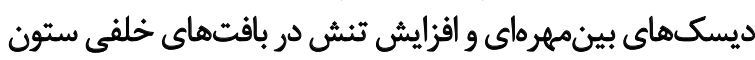

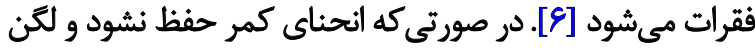

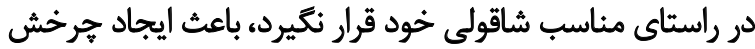

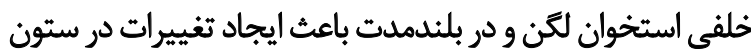

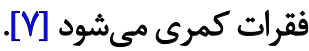

توانايى حفظ كنترل ياسجر يك عامل مهمه براي انجام بسيارى از

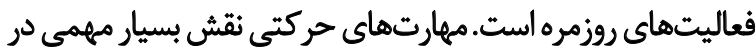

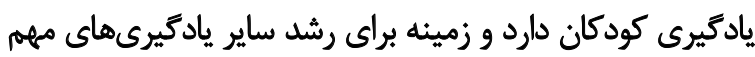

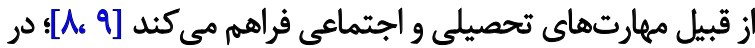

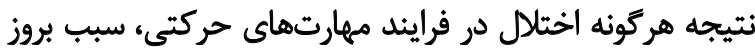

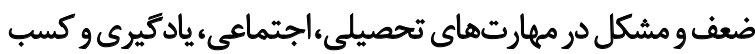

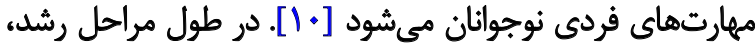

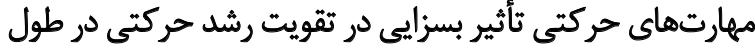

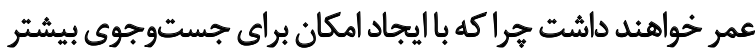

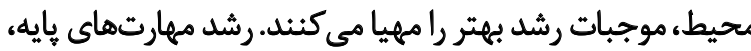

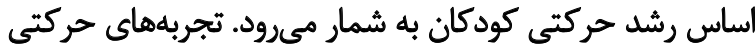

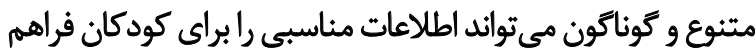

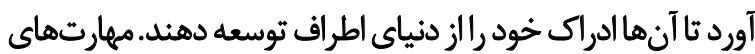

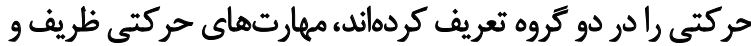

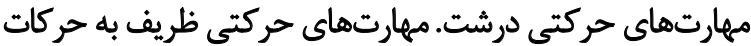

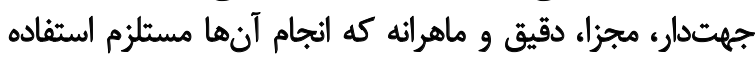

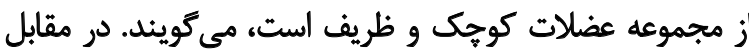

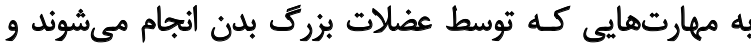

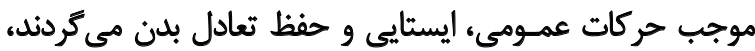

مهارتهاى حركتى درشت مي كويند [111]. كودك يس از رشد و يالايش الكوهاى حركتى بنيادى، از طريق 


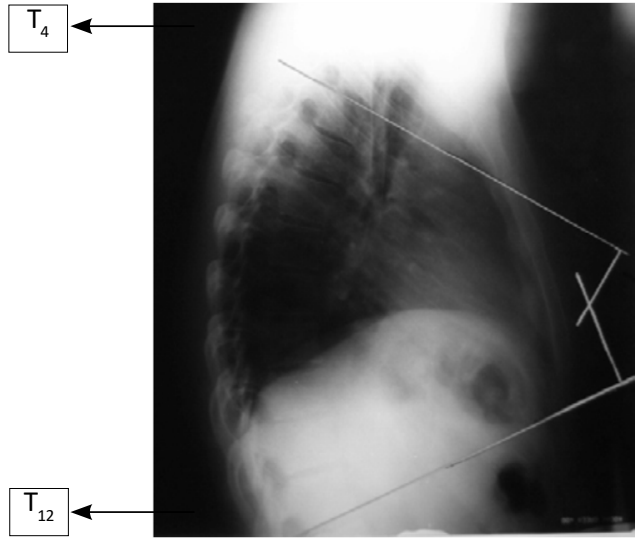

مجله بيومكانيك ورنثر

تصوير r. نحوه اندازهيرى قوسهاى ساجيتال ثبت شده از طريق روش كوب ميوميت

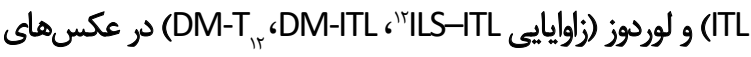
راديوكرافى أز روش كوب" استفاده شد.

زاويه به دست آمده از روش كوب متداولترين روشى است كه براى

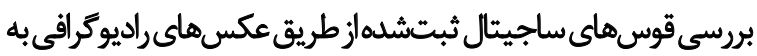

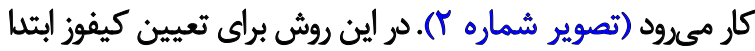

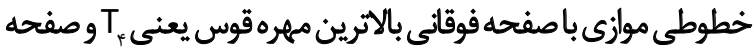

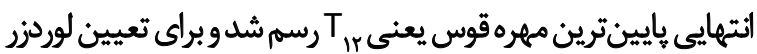

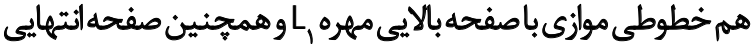

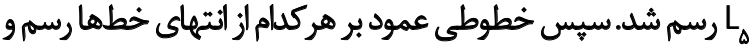

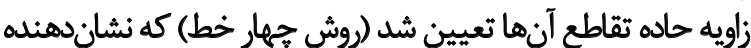

مقدار كيفوز ولوردوز است [1/ ا-19]]

ثتايج

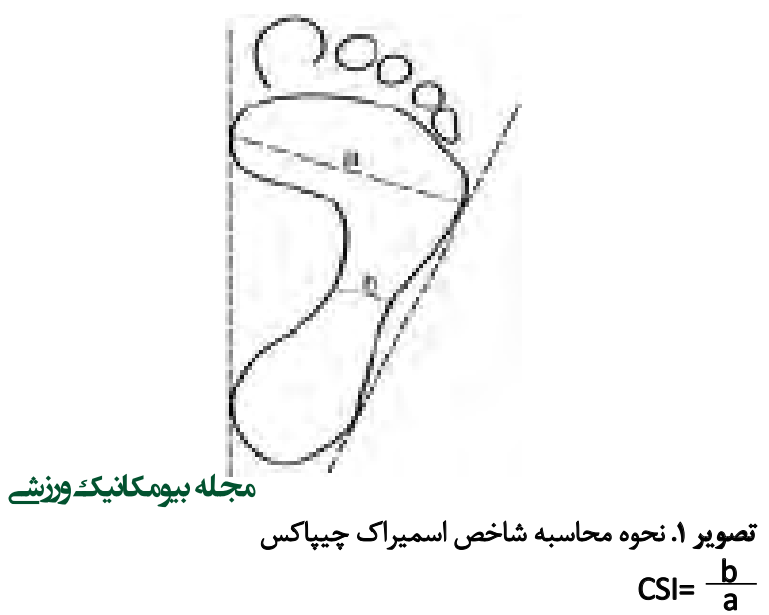

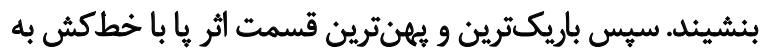

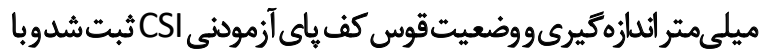

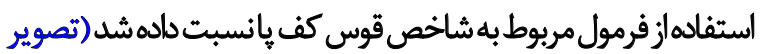

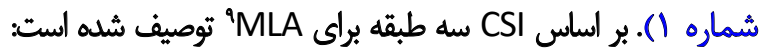

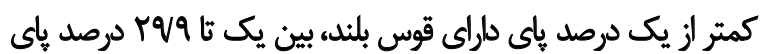

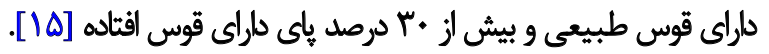

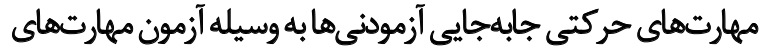

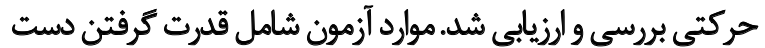

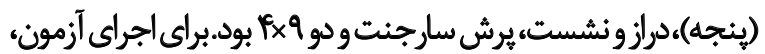

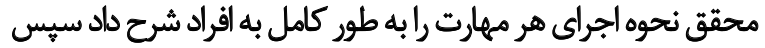

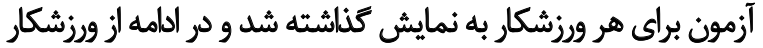

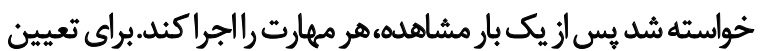

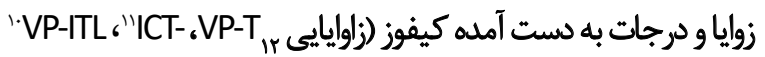

نتايج آزمون تى مستقل به منظور مقايسه متغيرهاى مهارت

9. Medial Longitudinal Arch (MLA)

10.Inclination Cervical Thoracic-Inclination Thoracic Lumbar

11. Vertebra prominens

12. Inclination lumbar sacral

13. Cobb angle

جدول ا. نتايج آزمون تى مستقل به منظور مقايسه متغيرهاى مهارت حركتى آزمودنىها در دو كروه واليبال و بسكتبال

\begin{tabular}{|c|c|c|c|c|c|c|}
\hline $\mathbf{P}$ & $\mathbf{t}$ & $\mathbf{F}$ & اختثلاف ميانكين & ميانكين ثانحراف استاندارد & كروه & متغيرها \\
\hline$. / p \mid$ & - /AP & $\Delta / M^{\prime}$ & V/AT & $\begin{array}{l}. / 0 T \pm .1 .8 \\
. / P V \pm . / 1\end{array}$ & بسكتبل & قدرت كرفتن دست (ينجه) \\
\hline . IVA & $-\cdot / M$ & $p / p q$ & $-1 / \cdot 9$ & $\begin{array}{l}\mathrm{rr} / \cdot V \pm V / \mathrm{V}^{\circ} \\
\mathrm{Pd} / \mathrm{V} \pm \mid \mathrm{V} / \mathrm{IP}\end{array}$ & واليبال & مراز و نشست (تعداد) \\
\hline$\%$ &.$/ T A$ & W/TI & $r+|8|$ & $\begin{array}{l}V \cdot / \& \mid \pm N Q \Lambda \\
V / \cdots \pm \& / Q \Lambda\end{array}$ & بسكتبال & يرش سارجنت (سانتىمت) \\
\hline$\%$ & - 119 & .1 .1 & $1 / 09$ & $\begin{array}{l}N N E \pm Y / F \Delta \\
q / N \pm q / N^{r}\end{array}$ & بسكتبال & دو \\
\hline
\end{tabular}

مجله بيومكانيك ورنثش 
جدول r. نتايج آزمون منويتنى به منظور مقايسه متغيرهاى ياسجر آزمودنىها در دو كروه واليبال و بسكتبال

\begin{tabular}{|c|c|c|c|c|c|}
\hline $\mathbf{P}$ & $\mathbf{Z}$ & ميانكين رتبه & ميانكين +انحراف استاندارد & كروه & شاخص \\
\hline .1119 & $-1 / T \Lambda$ & $\begin{array}{l}\text { IVMT } \\
\text { IF/Ma }\end{array}$ & $\begin{array}{l}I / R A \pm V / K \\
\cdot / V A \pm \cdot / 9 .\end{array}$ & واليبال & واروس \\
\hline . &.$- / 1 T$ & $\begin{array}{l}10 / r 0 \\
10 / 8 r\end{array}$ & $\begin{array}{l}\cdot(\Delta \mathrm{H} \pm 1 / \mathrm{m} \\
\cdot(\Delta \Delta \pm / / \mathrm{rr}\end{array}$ & بسكتبال & والكوس \\
\hline • & -+199 & $\begin{array}{l}18 / \pi 0 \\
18 / r k\end{array}$ & $\begin{array}{l}\cdot 1 \Delta \mathrm{H} \pm \cdot 188 \\
\cdot N \cdot \pm \cdot 18 \mathrm{~A}\end{array}$ & بسكتبال & يرونيشن \\
\hline $.1 \cdot \Delta 1$ & $-1 / 9 Y$ & $\begin{array}{l}\text { WNE } \\
\text { IF/TE }\end{array}$ & $\begin{array}{l}\cdot / 81 \pm \cdot 18 \Delta \\
\cdot / M T \pm \cdot 1 \Delta \theta\end{array}$ & واليبال & سويينيشن \\
\hline . & $-+/ M$ & $\begin{array}{l}1 \% / M \\
1 \% / 0\end{array}$ & $\begin{array}{l}\cdot / r \cdot \pm \cdot / P A \\
\cdot / R Y \pm \cdot / \Delta 1\end{array}$ & واليبال & لوردوز \\
\hline $.1+19$ & $-1 / 1 \Delta$ & $\begin{array}{l}\text { wre } \\
\text { ir/ra }\end{array}$ & $\begin{array}{l}. / 9 q \pm \cdot / \mathrm{ra} \\
\cdot / \mathrm{r} \pm \cdot / \mathrm{rw}\end{array}$ & واليبال & كايفوز \\
\hline - $r \Delta q$ & $-+/$ Af & $\begin{array}{l}|f / r| \\
\mid f / M\end{array}$ & $\begin{array}{l}. / 1 \Delta \pm . / m V \\
. / \cdot \Delta \pm . / \pi r\end{array}$ & بسكتبل & كف باي كود \\
\hline .1811 & $-1 / \pi r$ & $\begin{array}{l}\text { IV/Fe } \\
1 \% / \%\end{array}$ & $\begin{array}{l}. / 2 q \pm . / 01 \\
. / r q \pm \cdot / 81\end{array}$ & بسكيبال & كف هاي صاف \\
\hline
\end{tabular}

مجله بيومكانيكورنش

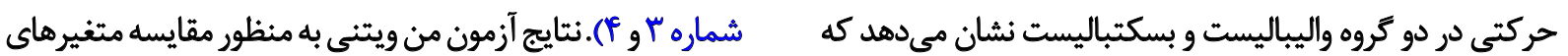

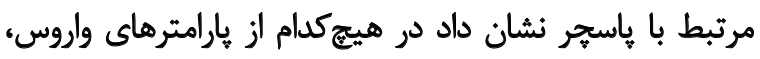

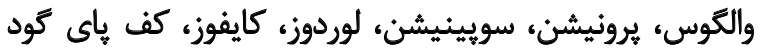

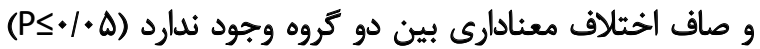

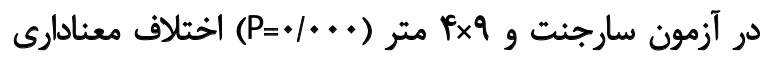

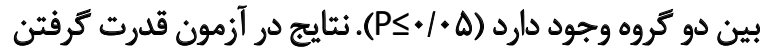

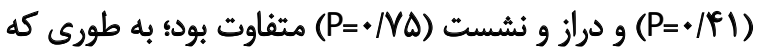
(جداول شماره ا و (T) (تصوير شماره هاف).

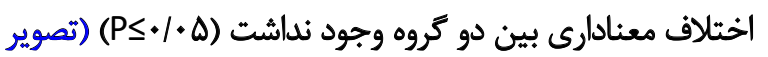
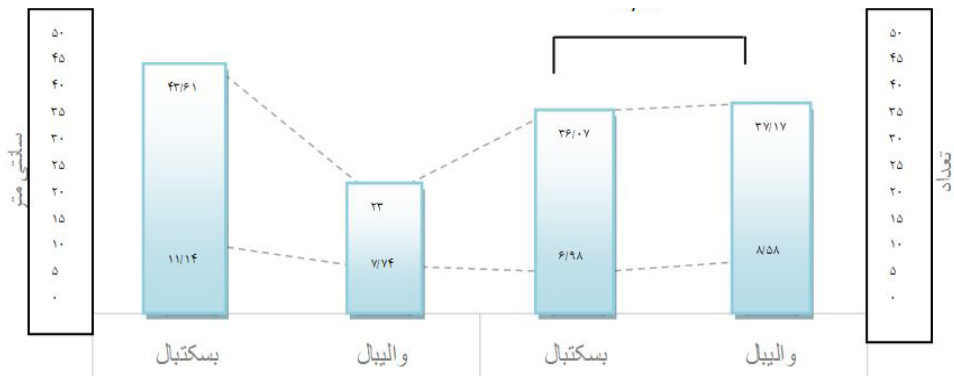

QSD QMean

\section{مجله بيومكانيكورزش}

تصوير ب. نتايج آزمون تى مستقل (يرش سارجنت و دراز ونشست) به منظور مقايسه متغيرهاى مهارت حركتى آزمودنىها در دو كروه واليبال و بسكتبال 


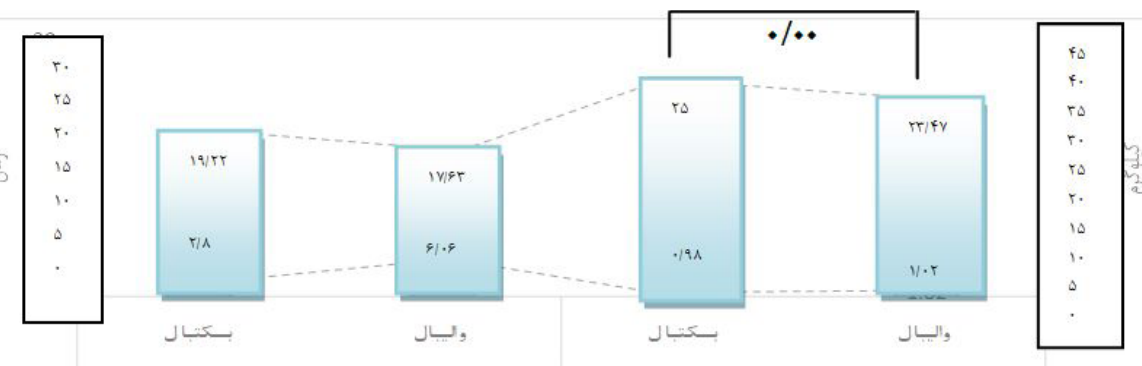

| 4×9

口SD $\square$ Mean

مجله بيومكانيك ورنشي

تصوير F. نتايج آزمون تى مستقل (دو qXX و قدرت كرفتن) به منظور مقايسه متغيرهاي مهارت حركتى آزمودنى ها در دو كروه واليبال و بسكتبال

نيز از ورزشكاران نخبه بودند، بنابراين شايد يكى از دلايل افزايش

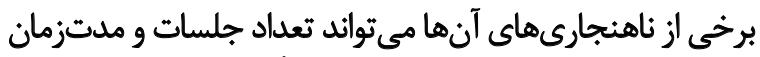

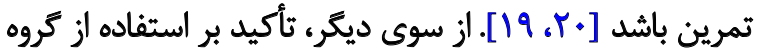
خاصى از عضلات كه در رشته مورد نظر كاربرد اختصاصى دارند و عدم توجه به عضلاتى كه در اجراى فنون نقش اصله اصلى ندارند

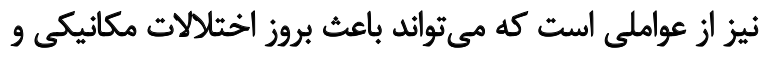

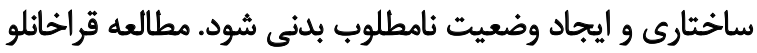

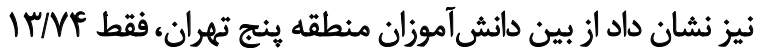

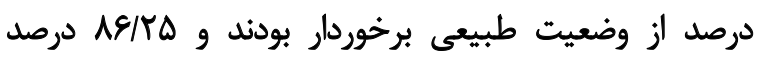

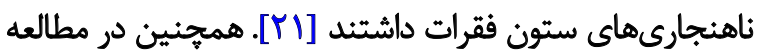

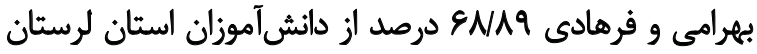

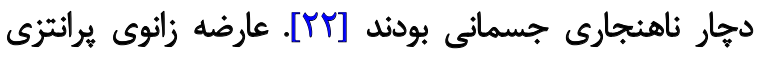

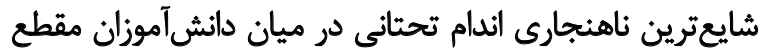

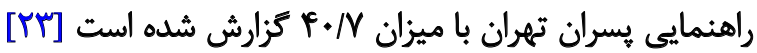

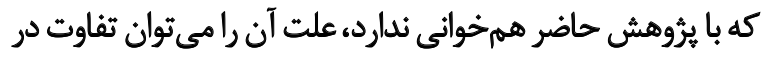

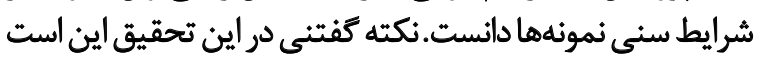

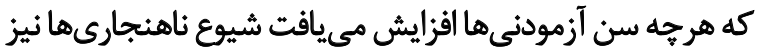

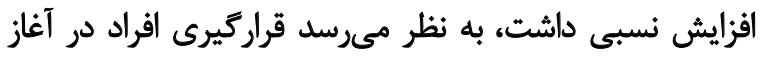

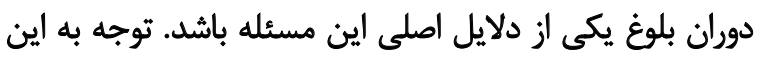

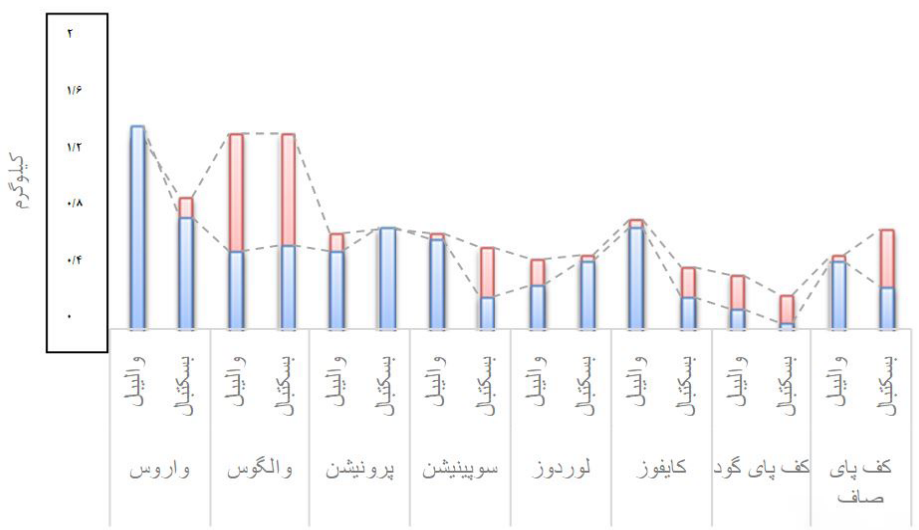
يروهش حاضر به مقايسه ميارتهاى حركتى منتخب (قدرت

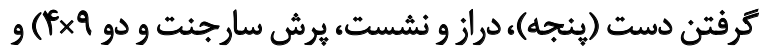

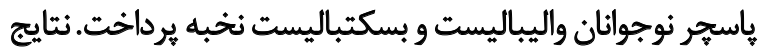

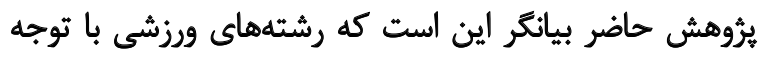

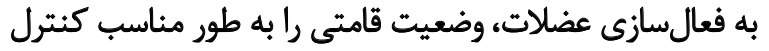

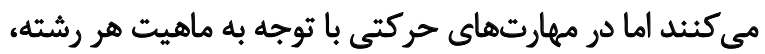

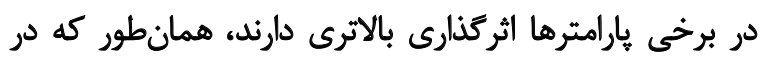

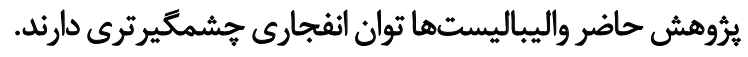

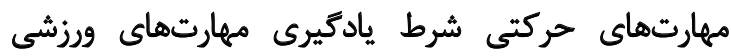

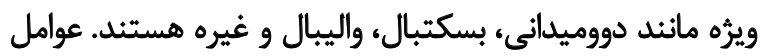

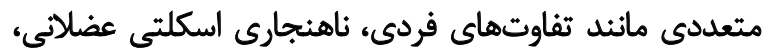

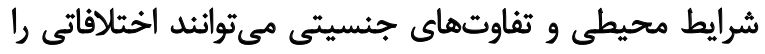

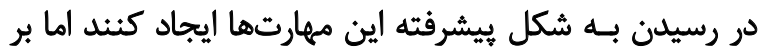

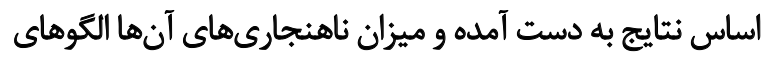

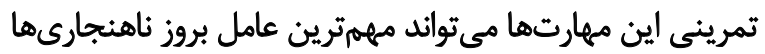

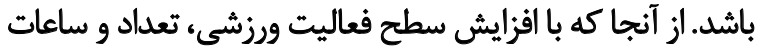

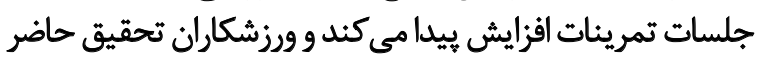

口SD QMean 


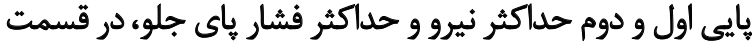

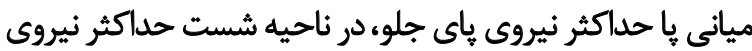

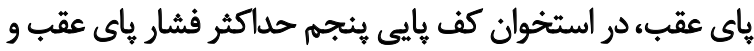

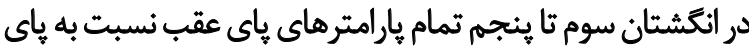

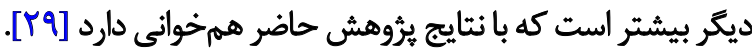

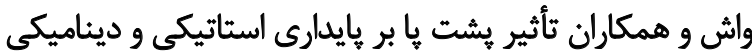

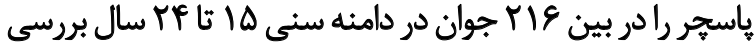

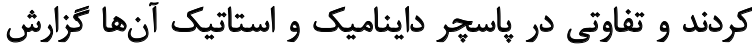

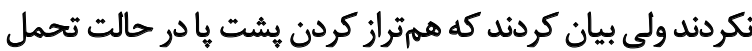

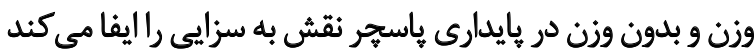

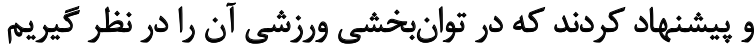

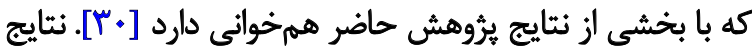

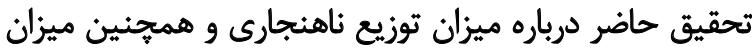

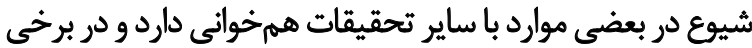

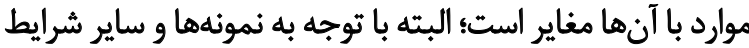

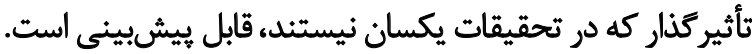

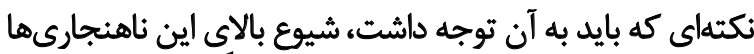

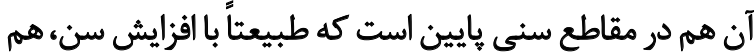
ميزان شيوع و هم شدت آنها آنزايش مي اسيابد.

\section{نتيجلكيرى نهايى}

وضعيت باسجر و آمادگى جسمانى ورزشكاران نقش به سزايى

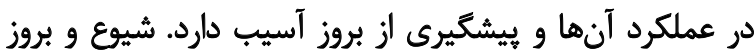

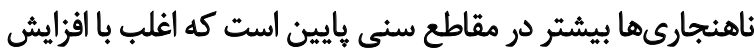

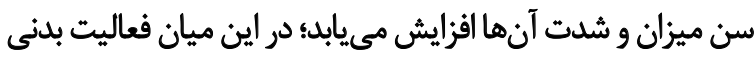

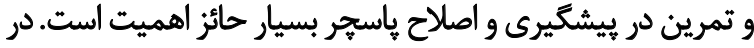

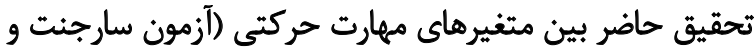

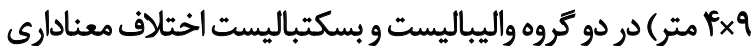

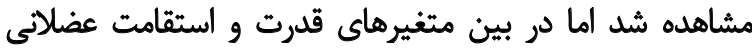

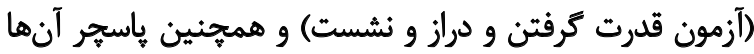

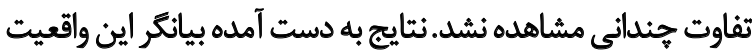

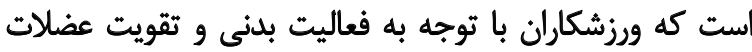

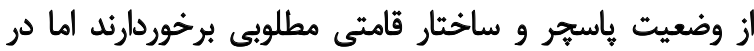

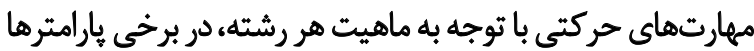

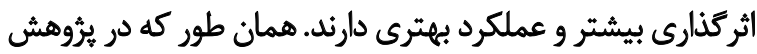

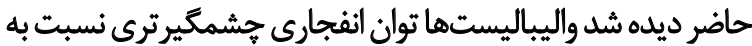

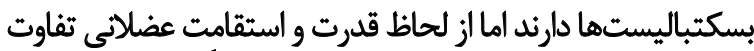

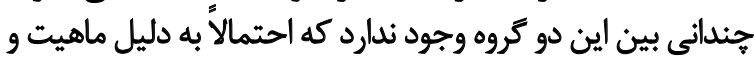

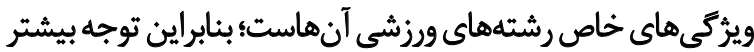

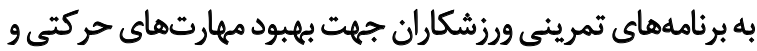

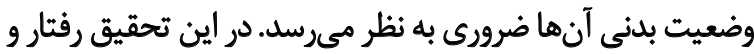

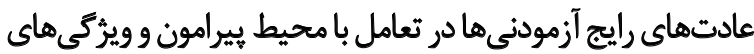

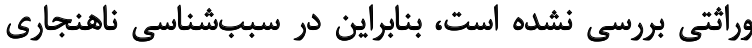
اسكلتى عضلانى به تحقيقات بيشترى نياز است.
نكته كه با افزايش سن، هم ميزان شيوع و هم شدت ناهنجاريها

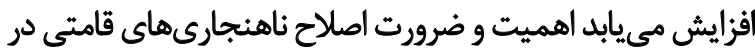

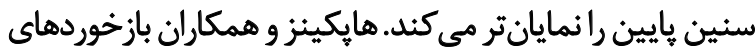

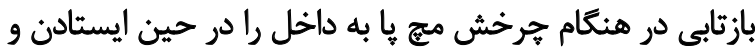

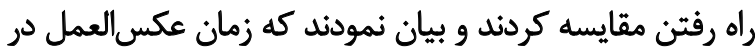

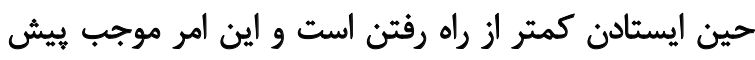

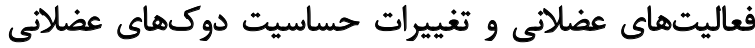

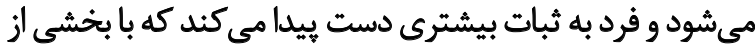

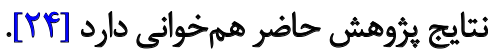
كوته و همكاران بيان كردند عملكرد تعادلى در شرايط ايستا

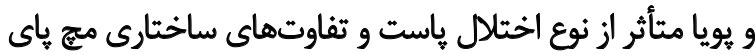

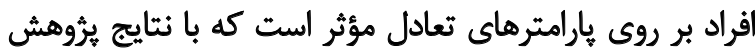

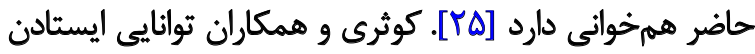

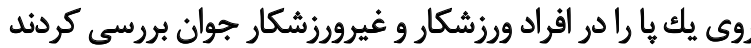

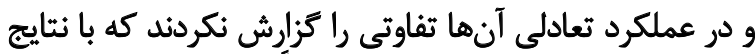

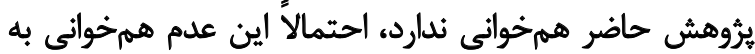

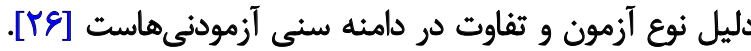

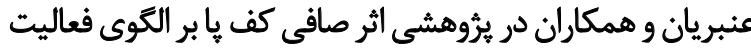

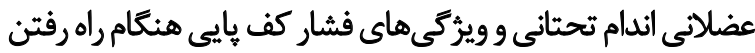

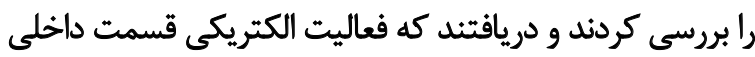

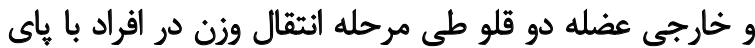

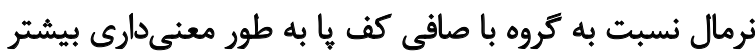

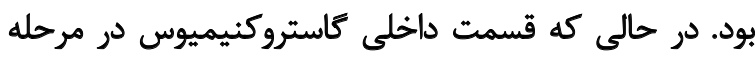

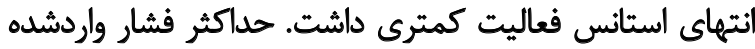

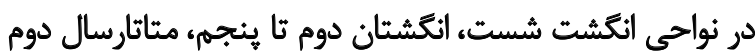

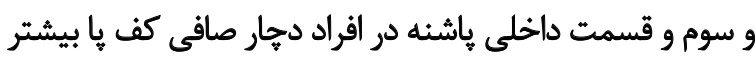

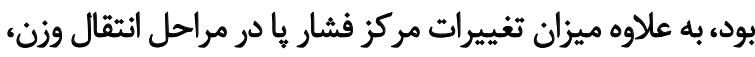

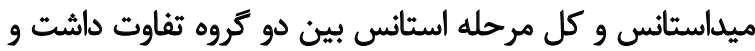

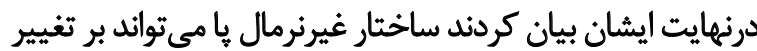

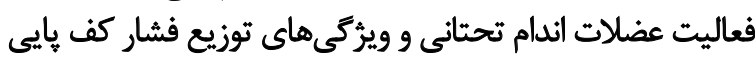

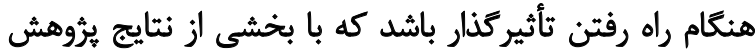

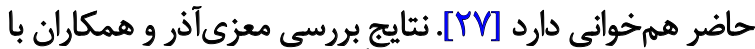

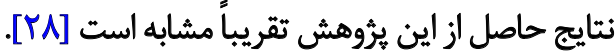

نتايج تحقيقات مختلف حاكى از بالا بودن ميزان ناهنجارىها

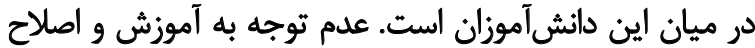

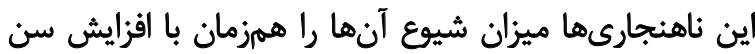

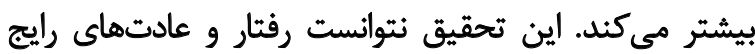

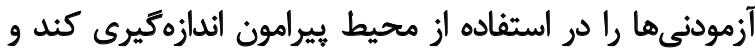

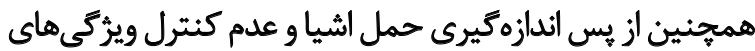

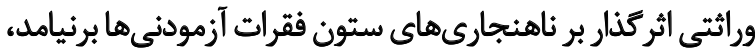

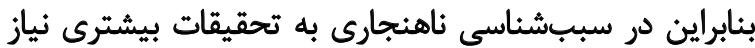

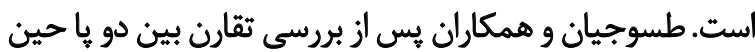

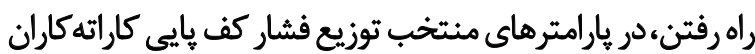

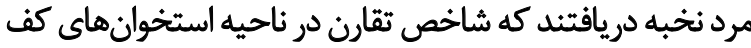




\section{ملاحظات اخلاقيى}

بيروى از اصول اخلاق يثوهش

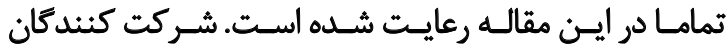

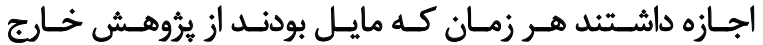

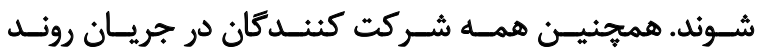

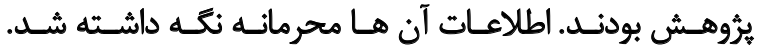

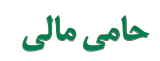

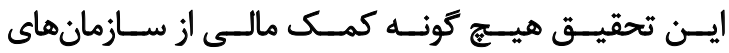

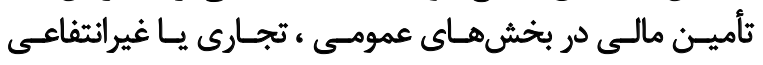

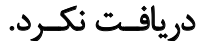

$$
\text { مشاركت نويسندكّان }
$$

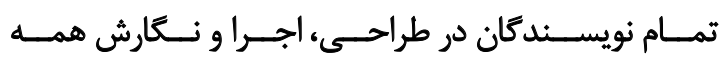

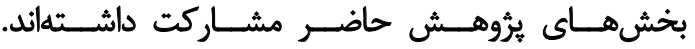

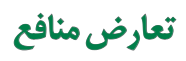

بنابر اظهار نويسندكان، اين مقاله تعارض منافع ندارد.

$$
\text { تشكر وقدردانى }
$$

نويسندكان ازمركز يويش ستاره سلامت تهران تشكر مى كنند. 


\section{References}

[1] Daneshmandi H, Alizadeh MH, Gharahkanloo R. Corrective exercises. $18^{\text {th }}$ edition. SAMT Publication; 2020. https://www.gisoom.com/ book/11661590/\%DA\%A9\%D8\%AA\%/

[2] Yousefi B. [The relationship between the desk and ergonomic characteristics anthropometric indicators of school students in Kermanshah city and skeletal anomalies of the spine and upper extremity (Persian)]. J Motion. 2005; 26(1):23-40. https://www.sid.ir/fa/journal/ViewPaper. aspx?id=41313

[3] Emery CA, Cassidy JD, Klassen TP, Rosychuk RJ, Rowe BB. Development of a clinical static and dynamic standing balance measurement tool appropriate for use in adolescents. Phys Ther. 2005; 85(6):502-14. [DOI:10.1093/ptj/85.6.502] [PMID]

[4] Zeighami S. [The role of exercise therapy and corrective movements in the treatment of musculoskeletal injuries in children and adolescents (Persian)]. Iran J Pediatr. 2002; 13(suppl). https://www.sid.ir/fa/Journal/ ViewPaper.aspx?ID=43538

[5] Rome K, Brown CL. Randomized Clinical trail into the impact of rigid foot orthoses on balance parameters in exercise sively pronated feet. Clin Rehabil. 2004; 18(6):624-30. [DOI:10.1191/0269215504cr767oa] [PMDI]

[6] Videman T, Nurminen M, Troup JD. 1990 Volvo Award in clinical sciences. Lumbar spinal pathology in cadaveric material in relation to history of back pain, occupation, and physical loading. Spine. 1990; 15(8):728-40. [DOI:10.1097/00007632-199008010-00002] [PMID]

[7] Endo K, Suzuki H, Nishimura H, Tanaka H, Shishido T, Yamamoto K. Sagittal lumbar and pelvic alignment in the standing and sitting positions. J Orthop Sci. 2012; 17(6):682-6. [DOI:10.1007/s00776-012-0281-1] [PMID]

[8] Seif S, Kadivar J. Specific learning disorder. Tehran: SAMT Publications; 1995.

[9] Baranek GT. Efficacy of sensory and motor interventions for children with autism. J Autism Dev Disord. 2002; 32(5):397-422. [DOI:10.1023/A:1020541906063] [PMID]

[10] Jepsen $\mathrm{RH}$, VonThaden $\mathrm{K}$. The effect of cognitive education on the performance of students with neurological developmental disabilities. Neuro Rehabilitation. 2002; 17(3):201-9. [DOI:10.3233/NRE-200217305] [PMID]

[11] Hasanati F, Khatoonabadi AR, Abdolvahab M. A comparative study on motor skills in 5-year-old children with phonological and phonetic disorders. Audiol. 2010; 19(1):71-7._https://citeseerx.ist.psu.edu/viewdoc/ download?doi=10.1.1.883.2437\&rep=rep1\&type=pdf

[12] Gallahue DL, Ozmun JC, Goodway J. Understanding motor development: Infants, children, adolescents, adults. Boston, MA: Mcgraw-hill; 2006.

[13] Abdoli B, Taymoori M, Zamani Sani SH, Zeraat Kar M, Hovnloo F. [Relationship between Plantar longitudinal arches and Some Selected Motor Parameters in Children aging 11 to 14 years (Persian)]. JRRS. 2011; 7(3):381-90. [DOI: 10.22122/jrrs.v7i3.209]

[14] Malina RA, Bouchard C. Growth, maturation and physical activity, $2^{\text {nd }}$ edition. Champaign, IL: Human Kinetics; 2004. [DOI:10.5040/9781492596837]

[15] Forriol F, Pascual J. Footprint analysis between three and seventeen years of age. Foot Ankle. 1990; 11(2):101-4. [DOI:10.1177/107110079 001100208] [PMID]
[16] Mannion AF, Knecht K, Balaban G, Dvorak J, Grob D. A new skin-surface device for measuring the curvature and global and segmental ranges of motion of the spine: Reliability of measurements and comparison with data reviewed from the literature. Eur Spine J. 2004; 13(2):122-36. [DOI:10.1007/s00586-003-0618-8] [PMID] [PMCID]

[17] Korovessis P, Petsinis G, Papazisis Z, Baikousis A. Prediction of thoracic kyphosis using the Debrunner kyphometer. J Spinal Disord. 2001; 14(1):67-72. [DOI:10.1097/00002517-200102000-00010] [PMID]

[18] Greendale GA, Nili NS, Huang MH, Seeger L, Karlamangla AS. The reliability and validity of three non-radiological measures of thoracic kyphosis and their relations to the standing radiological Cobb angle. Osteoporos Int. 2011; 22(6):1897-905. [DOI:10.1007/s00198-010-1422-z] [PMID] [PMCID]

[19] Fan Y, Fan Y, Li Z, Lv C, Luo D. Natural gaits of the non-pathological flat foot and high-arched foot. PloS One. 2011; 6(3):e17749. [DOI:10.1371/ journal.pone.0017749] [PMID] [PMCID]

[20] Wojtys EM, Ashton-Miller JA, Huston L, Moga PJ. The association between athletic training time and the sagittal curvature of the immature spine. Am J Sports Med. 2000; 28(4):490-8. [DOI:10.1177/0363546500 0280040801] [PMID]

[21] Parnow A, Qarakhanlu R, Parto, Aqa-Alinejad H. [Investigation of body composition profile, physiological, anthropometric of Iranian elite futsal players (Persian)]. J Phys Educ, Olympic. 2011; 13(2):49-58. https:// www.sid.ir/en/Journal/ViewPaper.aspx?ID=40275

[22] Bahrami M, Farhadi A. 9A survey of rate and causes of deformity in boys and girls youngsters in Lorestan province (Persian)]. Yafteh. 2007; 8(4):37-41. https://www.sid.ir/Fa/Journal/ViewPaper.aspx?id=60752

[23] Mirzaei R, Salimi N. [The study of height anomalies among secondary school students in Bayangan (Persian)]. Kermanshah Uni Med Sci. 2013; 16(7):e77297. https://sites.kowsarpub.com/jkums/articles/77297.html

[24] Hopkins JT, Palmieri R. Effect of Ankle joint effusion on lower leg functional. Clin J Sport Med; 2004; 14(1):1-7. [DOI:10.1097/00042752200401000-00001] [PMID]

[25] Cote KP, Brunet ME, Gansneder BM, Shultz SJ. Effects of pronated and supinated foot postures on static and dynamic postural stability. J Ath Train. 2005; 40(1):41-6. [PMID] [PMCID].

[26] Kosari S, Hemayat-Talab R, Arab-Ameri E, Keyhani F. The effect of physical exercise on the development of gross motor skills in children with attention deficit/hyperactivity disorder. Zahedan J Res Med Sci. 2013 15(2):e93113. https://sites.kowsarpub.com/zjrms/articles/93113.html

[27] Aminian G, Safaeepour Z, Farhoodi M, Pezeshk AF, Saeedi $H, M a-$ jddoleslam B. The effect of prefabricated and proprioceptive foot orthoses on plantar pressure distribution in patients with flexible flatfoot during walking. Prosthet Orthot Int. 2013; 37(3):227-32. [DOI:10.1177/0309364612461167] [PMID]

[28] Moezi Azar, Jali Shohreh Vaseghi, Gharamaleki Behnoosh. Prevalence of kyphosis and scoliosis in female middle school students in Tehran and the effect of sports activities and sitting position In their occurrence. Sci J Medical Organization Islam Repub Iran. 2014; 32(4):310-9. https://jmciri.ir/article-1-1727-fa.pdf

[29] Tasoujian E, Memar R. Survey symmetry in selected parameters of plantar pressure distribution in elite males karate athletes. J Res Sports Rehabil. 2017; 4(8):65-74. [DOI:10.22084/RSR.2017.7448.1139]

[30] Alaei B, Amiri Chayjan R, Sarikhani H. The effect of static and dy namic magnetic fields on some chemical properties of pomegranate arils. Iran J Biosys Engine. 2020; 50(4):823-31. [DOI:10.22059/IJBSE.2019.283275.665198] 
This Page Intentionally Left Blank 